\title{
Design and Discovery of Novel Curcumin-pyrazole-triazine Conjugates and its Evaluation against Multi-drug Resistant Microbes
}

\author{
Anjali $^{1 *}$, Udaya Pratap Singh ${ }^{2}$, P. Malairajan ${ }^{2}$, Rubina Lawrence ${ }^{1}$, Ebenezer Jeyakumar ${ }^{1}$ \\ ${ }^{1}$ Department of Industrial Microbiology, Jacob Institute of Biotechnology and Bioengineering \\ Sam Higginbottom University of Agriculture, Technology and Sciences, Prayagraj, India \\ ${ }^{2}$ Department of Pharmaceutical Sciences, Shalom Institute of Health and Allied Sciences, Sam Higginbottom University \\ of Agriculture, Technology and Sciences, Prayagraj, India
}

\begin{abstract}
*Address for Correspondence: Ms. Anjali, Department of Industrial Microbiology, Jacob Institute of Biotechnology and Bioengineering Sam Higginbottom University of Agriculture, Technology and Sciences, Prayagraj, Uttar Pradesh, India E-mail: anjali011189@gmail.com
\end{abstract}

Received: 13 Jan 2021/ Revised: 28 Mar 2021/ Accepted: 21 Jun 2021

\begin{abstract}
Background: In this work, unique curcumin pyrazole triazine conjugates were produced by combining curcumin with a nitrogencontaining heterocyclic compound 2, 4, 6-trichloro-1, 3, 5-triazine to create a physiologically relevant hybrid molecule capable of overcoming multi-drug resistance with improved pharmacological activity.

Methods: Synthesized drugs were characterized by Melting point, FTIR, ${ }^{1} \mathrm{H}$ NMR, ${ }^{13} \mathrm{C}$ NMR spectroscopy and Mass spectrometry. The conjugates were also evaluated for antibacterial (gram-positive and gram-negative bacteria) and antifungal (yeast and mould) activity with the help of the agar well diffusion method, the minimum inhibitory concentration via resazurin based micro-broth dilution method and minimum bactericidal concentration.

Results: The molecules containing halogens in their structure such as $A 5, A 8, A 3$, and $A 4$ have shown the best antibacterial with MIC ranging between $32-128 \mu \mathrm{g} / \mathrm{ml}$. Furthermore, the bioconjugate A5 had the most potent antibacterial action against Grampositive bacteria Staphylococcus aureus and Gram-negative bacterial Pseudomonas aeruginosa, as well as antifungal activity against Candida albicans.

Conclusion: Pharmaceutically important hybrid molecules can be used in future to cure bacterial and fungal diseases.
\end{abstract}

Key-words: Antibacterial activity, Antifungal activity, Curcumin conjugates, Multidrug resistance, Triazine conjugates

\section{INTRODUCTION}

Multidrug resistance (MDR) is a global health concern as drug-resistant pathogens "Superbugs" are continuously acquiring new strategies to become resistant ${ }^{[1]}$. According to the WHO, these resistant microbes (Bacteria, Fungi) can withstand antimicrobial drug attacks, resulting in inadequate medication, tolerance, and infection spread.

\section{How to cite this article}

Anjali, Singh UP, Malairajan P, Lawrence R, Jeyakumar E. Design and Discovery of Novel Curcumin-pyrazole-triazine Conjugates and its Evaluation against Multi-drug Resistant Microbes. SSR Inst. Int. J. Life Sci., 2021; 7(4): 2834-2843.

\begin{tabular}{|c|c|}
\hline & Access this article online \\
\hline & https://ijls.com/
\end{tabular}

Exposure to these medications creates the requisite selective pressure for the emergence and dissemination of resistant pathogens ${ }^{[2]}$.

Despite renewed attempts to produce novel antibiotics, the number of medication approvals has been steadily declining recently due to their major ineffectiveness towards MDRs ${ }^{[3-5]}$. As a result of these concerns, there has increased interest in research into alternative antimicrobials. Measurable studies highlighted the role of natural compounds and their extracts in reducing antibiotic dependence in bacterial treatment, thus avoiding the development of antibiotic resistance ${ }^{[6]}$. These cost-effective natural compounds are now understood to have various biological, chemical, and physical functions, and those plants such as turmeric 
(curcumin), clove, allspice, cinnamon, thyme, and garlic contain numerous antimicrobial compounds ${ }^{[7-9]}$.

Distinct molecular diversity of these natural products can be used to enhance their intrinsic biological activity or drug-like properties by designing combinatorial libraries. Even after their effective tolerability with less or negligible toxicity, their limitations in physicochemical properties making them barely dissolved in gastrointestinal aqueous fluid ${ }^{[10,11]}$. This can be accomplished by semi-synthetic modification of the parent molecule after critical structural elements necessary for biological activity are identified in libraries and validated using computing methods ${ }^{[12]}$. This approach of conjugation with other materials enhanced the biological activity of natural compounds such as ADMET (Adsorption, Distribution, Metabolism, Excretion and Transport), the physicochemical properties once administered in the human's body ${ }^{[13]}$.

With the simplicity of 2, 4, 6-trichloro-1, 3, 5-triazine conjugates with its ability to promote biological activities against multidrug-resistant bacteria, research on these (s-triazine) heterocyclic compounds has proven to be an efficient lead compound. The s-triazine compound may be a good economically viable starting material for developing a new antibiotic conjugating form ${ }^{[14]}$. It has been widely studied that s-triazine has high antibacterial and antifungal efficacy, which is primarily produced by a nucleophilic substitution reaction ${ }^{[15]}$. And for the first time, this economically feasible starting material was conjugated to an ingredient (curcumin) of the prestigious natural product Turmeric in the hope of improving synergistic anti-MDR activity and to some extent good physicochemical properties.

\section{MATERIALS AND METHODS}

Chemicals used for research works were procured from Rankem Chemical, India. Whattmann Filter paper no. 1 and 2,4,6-trichloro1,3,5-triazine (Sigma Aldrich). A thin layer chromatography plate was prepared by using silica gel-G on a glass plate. The spots were examined in an iodine chamber. Melting points of products as well as intermediates was examined through the Gallenkamp melting point apparatus (HICON, India) and were uncorrected. The FTIR spectra (in $2.0 \mathrm{~cm}^{-1}$, flat, smooth, abex) were recorded on the Perkin Elmer-Spectrum RX-I spectrophotometer. The ${ }^{1} \mathrm{H} N M R$ and ${ }^{13} \mathrm{C}$ NMR spectra were recorded on Bruker Advance II 400 and 100 NMR spectrophotometer. A mass spectrum was recorded on
THERMO-TSQ 8000. Varied multidrug-resistant grampositive bacteria S. aureus (MCCB0017), S. pyogenes (MCCB0093), Bacillus cereus (MCCB0143), B. subtilis (MCCB0189), C. perfringens (MCCBR040), L. monocytogenes (MCCB0028) and gram negative bacteria E. coli (МССВ0016), Salmonella typhi (МССВ0127), S. dysenteriae (МССВ0128), V. cholera (МССВ0047), C. jejuni (MCCB0303), H. pylori (MCCB0301, P. aeruginosa (МСCB0035) were selected for biological evaluation of drugs. Pathogenic fungi such as C. albicans (MCCB0290) and $A$. fumigatus (MCCB0206) were also selected for biological evaluation.

\section{General procedure for synthesis of curcumin conjugates and its characterization}

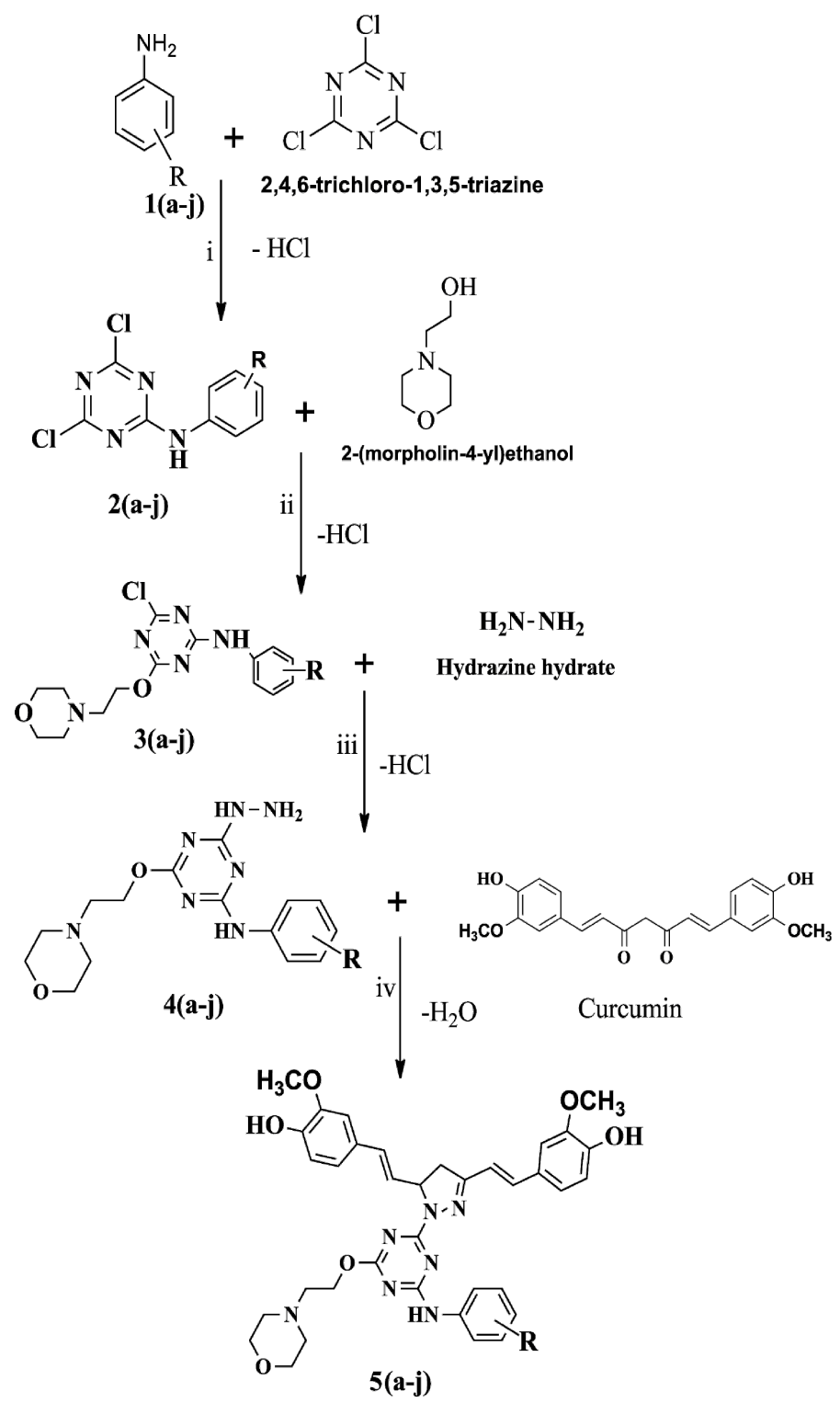

Fig. 1: Scheme of Curcumin pyrazole triazine conjugates synthesis 
Where (a-j) : $a=C N . \mathrm{SO}_{4}, \mathrm{~b}=\mathrm{CN}, \mathrm{c}=3-\mathrm{F}, \mathrm{d}=4-\mathrm{Br}, \mathrm{e}=3-\mathrm{Cl}, \mathrm{f}$ $=3-\mathrm{NO}_{2}, \mathrm{~g}=2-\mathrm{NO}_{2}, \mathrm{~h}=2-\mathrm{Cl}, \mathrm{i}=4-\mathrm{NO}_{2}, \mathrm{j}=\mathrm{H}$

Reagents and Reaction conditions-

(i) = Acetone medium, Sodium bicarbonate at $0-5^{\circ} \mathrm{C}$.

(ii) = Acetone medium, Sodium hydroxide at $45-50^{\circ} \mathrm{C}$

(iii)= Acetone medium, sodium hydroxide at $60^{\circ} \mathrm{C}$

(iv) $=$ Glacial acetic acid, reflux temperature below $100^{\circ} \mathrm{C}$

Synthesis of $\mathbf{N}$ substituted phenyl-1, 3, 5-triazine-2amine derivatives as compound (2a-j)- Reaction as shown in Fig. 1 between 2, 4, 6-trichloro-1, 3, 5-triazine $(0.01 \mathrm{M})$ and $1(\mathrm{a}-\mathrm{j})(0.01 \mathrm{M})$ was carried out by stirring in presence of $(20 \mathrm{ml})$ acetone at $0-5^{\circ} \mathrm{C}$. Meanwhile, (0.1M) $\mathrm{NaHCO}_{3}$ used to neutralize liberated $\mathrm{HCl}$. Completion of the reaction was verified by TLC (acetone: benzene, 1:1). The addition of crushed ice resulted in the precipitate and then filtered. The purity of the compound was checked by using a melting point. The product was airdried $^{[16]}$.

Synthesis of 4-chloro-6-(2-morpholinoethoxy)-N-Rphenyl-1,3,5-triazin-2-amine derivatives as compound (3a-j)- Equimolar amount (0.01M) of product 2(a-j) and 2-(moroholin-4-yl) ethanol as shown in Fig. 1 was taken in a three-necked round bottom flask fitted with the water condenser. The mixture was refluxed at $45-50^{\circ} \mathrm{C}$ in presence of acetone. Here, (0.1M) NaOH used for the neutralization of liberated acid. Completion of the reaction was confirmed by TLC (acetone: benzene, 2:1) and the visualization of the compound was carried out in an iodine chamber, after that re-crystallization done by ethanol only in the case of 4-aminobenzonitrile containing derivatives. Purity of compounds determined by the measuring melting point, after filtration, the product was vacuum dried ${ }^{[17]}$.

Synthesis of $\mathrm{N}$ substituted phenyl-4-hydrazinyl-6-(2morpholinoethoxy)-1, 3, 5-triazin-2-amine derivatives as compound (4a-j).

In this step the intermediate product 3 (a-j) (0.01M) refluxed with hydrazine hydrate $(0.01 \mathrm{M})$ as shown in Fig. 1 in presence of acetone $(20 \mathrm{ml})$ was taken in a threenecked round bottom flask fitted with the water condenser. Basicity was maintained (0.1M) $\mathrm{NaOH}$. Completion of the reaction was monitored by TLC with appropriate solvent systems. The visualization of the compound was carried out in an iodine chamber. The product was purified by re-crystallization by using ethanol in the case of 4-aminobenzonitrile containing triazine. Thereafter compound was filtered and vacuum dried.

Synthesis of of $\mathrm{N}$-phenylamino -1, 3, 5-triazin-2-yl)-4, 5dihydro-1H-pyrazol-3-yl) vinyl)-2-methoxyphenol derivatives as compound $\mathbf{5}(\mathbf{a}-\mathbf{j})-$ In the final step as shown in Fig. 1 above product 4(a-j) (0.01M) was conjugated to 1,7-bis(4-hydroxy-3-methoxyphenyl) hepta-1,6-diene-3,5-dione (curcumin, 0.01M) at reflux temperature in presence of glacial acetic acid $(30 \mathrm{ml})$. Completion of the reaction was monitored by the TLC (acetone: benzene, 1:1). The visualization of the compound was carried out in an iodine chamber. The product was re-crystallized with ethanol only in the case of 4-aminobenzonitrile containing triazine. After filtration of the compound, it was dried in a vacuum. The purity of the compound was checked by determining the melting point ${ }^{[18]}$.

Antibacterial activity- Different conjugates were screened for antibacterial activity according to the guidelines of the Clinical Laboratory Standard Institute (CLSI). Different pathogenic strains of gram-negative bacteria and gram-positive bacteria were used for screening. The experiment was conducted using nutrient broth media. The nutrient broth culture with loopful bacterial strains was incubated at $37 \pm 1^{\circ} \mathrm{C}$ for $16-18 \mathrm{hrs}$, and the microbial culture was adjusted to the McFarland standard. Diluting the bacterial suspension with sterile solution yielded a final concentration of $1.5 \times 10^{8} \mathrm{CFU} / \mathrm{mL}$. The plates of nutrient agar media were prepared. With the help of stainless steel cork, 5-mm diameter wells were made into swabbed agar plates. To make a neat solution, testing compounds were dissolved in dimethyl sulfoxide (DMSO). Following that, the wells were loaded with $30 \mu$ of testing samples and incubated at $37 \pm 1^{\circ} \mathrm{C}$ for 16-18 hrs and it was performed in triplicate. Their activity was assessed by measuring the zone of inhibition against bacterial pathogens using a zone reader (Himedia zone scale) $^{[19]}$.

Minimum inhibitory concentration (MIC) and Minimum bactericidal concentration (MBC)- Different bacterial strains were screened for MIC. Each well of a 96-well microplate was filled with $75 \mu \mathrm{l}$ nutrient broth and for testing drugs of many concentrations in 2-fold dilution as $1024,512,256,128,64,32,16 \mu \mathrm{g} / \mathrm{ml}$. Then $75 \mu \mathrm{l}$ of each test organism was inserted in each well. The remaining 
Cross ref DOI: 10.21276/SSR-IIJLS.2021.7.4.2

two wells in each row of microplates were then allowed to go negative control (i.e., the extract was substituted with $50 \mu \mathrm{l}$ of $10 \%$ DMSO) and positive control as no extract but $50 \mu$ lantibiotic. Finally, each well-received 50 $\mu \mathrm{l}$ of resazurin solution. The experiments were conducted in triplets and bacterial isolates were incubated for $24 \mathrm{~h}$ at $37 \pm 1^{\circ} \mathrm{C}$. The last volume of a drug capable of penetrating visible microbial growth of microorganisms or no change in resazurin dye colour was then reported as the MIC value ${ }^{[20]}$. To assess MBC, a loopful of the mixture of each well that did not exhibit microbial growth was sub-cultured by streaking on nutrient agar plates and incubated for $24 \mathrm{~h}$ at $37 \pm 1^{\circ} \mathrm{C}$. $\mathrm{MBC}$ was recorded as the lowest concentrations of extract that did not display any established colony ${ }^{[21]}$.

Antifungal activity- This method was performed according to the guidelines of CLSI. Sabouraud dextrose agar/broth media for $C$. albicans and potato dextrose agar for $A$. fumigatus were used to experiment. Broth culture with a loopful fungal strain was incubated at $37 \pm 1^{\circ} \mathrm{C}$ for $16-18 \mathrm{hrs}$ for yeast and $28 \pm 1^{\circ} \mathrm{C}$ for $72 \mathrm{hrs}$ mold and microbial culture was adjusted to McFarland standard for yeast $1.5 \times 10^{8}$ and spore suspension adjusted for mold at $5 \times 10^{4}$ spores. Total $20 \mathrm{ml}$ media were poured then solidifying plates were swabbed using a sterilized cotton swab with $100 \mu$ l. With the help of a cork borer of $5 \mathrm{~mm}$ diameter wells were made. Testing compounds were dissolved in DMSO to make a neat solution. After that the wells were loaded with $30 \mu \mathrm{l}$ of testing samples and allowed to incubate at $37 \pm 1^{\circ} \mathrm{C}$ for 16-18 hrs for yeast and $28 \pm 1^{\circ} \mathrm{C}$ for $72 \mathrm{hrs}$ mold. Their activity was evaluated by measuring the zone of inhibition. The procedure was performed in triplicate for the pathogen. The compound was tested against the test organism in triplicate ${ }^{[19] .}$

Statistical Analysis- The antibacterial activity of different curcumin conjugates synthesized in the present study was analyzed using two-way Analysis of Variance (ANOVA) followed by F-test and the significance was tested at $5 \%, 1 \%$ and $0.1 \%$ and results interpreted accordingly.

\section{RESULTS}

Chemistry and characterization of synthesized compounds- The designed library of target compounds and respective intermediates were synthesized as outlined in Fig. 1. Aromatic amines (4-aminobenzonitrile, 4-nitroaniline, 3-nitroaniline, 3-floroaniline, 4bromoaniline, 3-chloroaniline, 2-niroaniline, 2chloroaniline, and aniline) and 2, 4, 6, - trichloro-striazine are the reactants involved in the first step in the scheme for the synthesis of curcumin conjugates. The formation of mono substituted triazine occurred as a result of a nucleophilic aromatic substitution reaction with the hydrolysis of one of the chloro groups in the form of $\mathrm{HCl}$ in the presence of acetone as a solvent and sodium bicarbonate $\left(\mathrm{NaHCO}_{3}\right)$ as a neutralizing agent. In the second step an intermediate $2(a-j)$ reacted with 2(morpholin-4-yl) ethanol, where $\mathrm{OH}$ group of morpholine hydrolyzed with one of the chloro group via nucleophilic substitution reaction of s-triazine to form di-substituted s-triazine derivative. This reaction took place in the presence of acetone and liberated acid $(\mathrm{HCl})$, which was neutralized by sodium hydroxide $(\mathrm{NaOH})$. The second intermediate $3(a-j)$ of the next step, then reacted with hydrazine hydrate to form tri substituted 1, 3, 5-triazine with $\mathrm{HCl}$ liberation. In the last step, curcumin conjugated to $4(a-j)$ intermediate that resulted in the cyclization of diketone moiety of curcumin by hydrazine hydrate with the liberation of a water molecule to form final product as N-phenyl-4-hydrazinyl-6-)2- morpholinoethoxy)-1,3,5triazine-2-amine derivatives. The final compounds were characterized by various spectroscopic data's such as FTIR, ${ }^{1} \mathrm{H}$ NMR, ${ }^{13} \mathrm{C}$ NMR and Mass spectra.

\section{4-((4-(3,5-bis(4-hydroxy-3-methoxyphenethyl)-4,5-} dihydro-1Hpyrazol-1-yl)-6 (2morpholinoethoxy)-1,3,5triazin-2-yl)amino)benzonitrile sulfate hydrate. A2Yellow amorphous solid; Yield: 40\%; M.P.:210 $\mathrm{C}$ FTIR (vmax; cm ${ }^{-1} \mathrm{KBr}$ ): 3196.38 (O-H stretching), $3080.37(\mathrm{~N}-\mathrm{H}$ stretching), 2923.38 (Aromatic $\mathrm{C}-\mathrm{H}$ stretching), 2230.42 $\left(\mathrm{OCH}_{3}\right.$ stretching), 1738.28 ( $\mathrm{C}=\mathrm{O}$ stretching), 1683.31 ( $\mathrm{C}=\mathrm{N}$ stretching), $1586.20\left(\mathrm{CH}_{2}\right.$ bending), $1551.21 \quad(\mathrm{C}=\mathrm{C}$ stretching), 1367.27 ( $\mathrm{N}-\mathrm{N}$ stretching), 779.42; ${ }^{1} \mathrm{H} \mathrm{NMR}$ (400MHz, DMSO-d6, TMS) $\delta$ ppm: 10.84 (s, 2H, Ar-OHx2), 7.46 (d, 2H, J=0.01 Hz, Ar-H), 7.32 (d, 2H, J=0.71 Hz, Ar$\mathrm{H}), 6.81(\mathrm{~d}, 1 \mathrm{H}, \mathrm{J}=0.78 \mathrm{~Hz}$, pyrazole-H), 6.77 (d, 2H, J=0.01 $\mathrm{Hz}, \mathrm{Ar}-\mathrm{H}), 6.73$ (d, 2H, J=0.78 Hz, Ar-H), 6.57 (d, 2H, $\mathrm{J}=0.72 \mathrm{~Hz}, \mathrm{Ar}-\mathrm{H}), 3.83(\mathrm{~s}, 1 \mathrm{H}, \mathrm{NH}), 3.68\left(\mathrm{~s}, 6 \mathrm{H}, \mathrm{OCH}_{3} \times 2\right)$, 3.60 (t, $2 \mathrm{H}, \mathrm{J}=3.47 \mathrm{~Hz}, \mathrm{CH}_{2}$, methylene), $3.58(\mathrm{~d}, 4 \mathrm{H}$, $\mathrm{J}=0.14 \mathrm{~Hz}$, morpholine-H), 2.51-1.91 (m, 4H, aliphatic $\mathrm{CHx} 4$ ), 2.49 (t, 2H, J=3.60 Hz, $\mathrm{CH}_{2}$, methylene), 2.50 (d, $4 \mathrm{H}, \mathrm{J}=3.60 \mathrm{~Hz}$, morpholine-H); ${ }^{13} \mathrm{C}$ NMR (100MHz, DMSOd6) $\delta$ ppm:172.07, 165.52, 164.39, 161.36, 148.19, 133.07, 132.73, 124.07, 120.57, 119.04, 113.28, 108.87, 
$108.73,107.86,104.95,40.03,39.82,39.62,39.41$, $39.20, \quad 38.99, \quad 38.78, \quad 21.40, \quad 21.04$; GC-MS: 281.0 (Triazine-Morpholine-M), 326.5 (Triazine- morpholine and amine, $\mathrm{M}+\mathrm{H}$ ).

\section{4-((4-(3,5-bis(4-hydroxy-3-methoxyphenethyl)-4,5-di} hydro-1Hpyrazol-1-yl)-6-(2-morpholinoethoxy)-1,3,5-tri azin-2-yl)amino)benzonitrile. A2- Dark brown amorphous solid; Yield: 44\%; M.P.:180 ${ }^{\circ}$ C; FTIR (vmax; $\mathrm{cm}$ $1 \mathrm{KBr}): 3196.38$ (O-H stretching), $3080.37 \quad(\mathrm{~N}-\mathrm{H}$ stretching), 2923.38 (Aromatic C-H stretching), 2230.42 $\left(\mathrm{OCH}_{3}\right.$ stretching), 1738.28 ( $\mathrm{C}=\mathrm{O}$ stretching), 1683.31 ( $\mathrm{C}=\mathrm{N}$ stretching), $1586.20\left(\mathrm{CH}_{2}\right.$ bending), $1551.21 \quad(\mathrm{C}=\mathrm{C}$ stretching), 1367.27 (N-N stretching), 779.42; ${ }^{1} \mathrm{H}$ NMR (400MHz, DMSO-d6, TMS) $\delta$ ppm: 10.84 (s, 2H, Ar-OHx2), 7.46 (d, 2H, J=0.01 Hz,Ar-H), 7.32 (d, 2H, J=0.71 Hz, Ar-H), $6.81(\mathrm{~d}, 1 \mathrm{H}, \mathrm{J}=0.78 \mathrm{~Hz}$, pyrazole-H), $6.77(\mathrm{~d}, 2 \mathrm{H}, \mathrm{J}=0.01$ $\mathrm{Hz}, \operatorname{Ar}-\mathrm{H}), 6.73(\mathrm{~d}, 2 \mathrm{H}, \mathrm{J}=0.78 \mathrm{~Hz}, \operatorname{Ar}-\mathrm{H}), 6.57$ (d, $2 \mathrm{H}$, $\mathrm{J}=0.72 \mathrm{~Hz}, \mathrm{Ar}-\mathrm{H}), 3.83(\mathrm{~s}, 1 \mathrm{H}, \mathrm{NH}), 3.68\left(\mathrm{~s}, 6 \mathrm{H}, \mathrm{OCH}_{3} \times 2\right)$, $3.60\left(\mathrm{t}, 2 \mathrm{H}, \mathrm{J}=3.47 \mathrm{~Hz}, \mathrm{CH}_{2}\right.$, methylene), 3.58 (d, $4 \mathrm{H}$, $\mathrm{J}=0.14 \mathrm{~Hz}$, morpholine- $\mathrm{H}), 2.51-1.91(\mathrm{~m}, 4 \mathrm{H}$, aliphatic $\mathrm{CH} 4$ ), 2.49 (t, $2 \mathrm{H}, \mathrm{J}=3.60 \mathrm{~Hz}, \mathrm{CH}_{2}$, methylene), 2.50 (d, $4 \mathrm{H}, \mathrm{J}=3.60 \mathrm{~Hz}$, morpholine-H); ${ }^{13} \mathrm{C}$ NMR (100MHz, DMSOd6) $\delta$ ppm:172.07, 165.52, 164.39, 161.36, 148.19, $133.07,132.73$, 124.07, 120.57, 119.04, 113.28, 108.87, $108.73,107.86,104.95,40.03,39.82,39.62,39.41$, $39.20,38.99, \quad 38.78, \quad 21.40,21.04$; GC-MS: 281.0 (Triazine-Morpholine-M), 326.5 (Triazine-morpholine and amine, $\mathrm{M}+\mathrm{H}$ ).

4-(2-(1-(4-((4-f luorophenyl)amino)-6-(2- morpholino ethoxy)-1,3,5-triazin-2-yl)-3(4-hydroxy-3-methoxystyryl) -4,5-dihydro-1Hpyrazol-5-yl)ethyl)-2-methoxyphenol.

A3- Dark brown amorphous solid; Yield: 67.05\%; M.P.:58 ${ }^{\circ} \mathrm{C} ;$ FTIR (vmax; $\mathrm{cm}^{-1} \mathrm{KBr}$ ): $3367.80 \quad$ (O-H stretching), $1579.51 \quad\left(\mathrm{C}=\mathrm{N}\right.$ stretching), $1506.50 \quad\left(\mathrm{CH}_{2}\right.$ bending), 1395.27 (N-N stretching), 1262.78, 1124.04 (CF stretching), 906.25, 608.10; ${ }^{1} \mathrm{H}$ NMR $(400 \mathrm{MHz}$, DMSOd6, TMS) $\delta$ ppm: 8.08 (s, 2H, Ar-OHx2), 7.63 (d, $2 \mathrm{H}$, $\mathrm{J}=1.43 \mathrm{~Hz}, \mathrm{Ar}-\mathrm{H}), 7.46$ (d, 2H, J=1.55 Hz, Ar-H), $7.14(\mathrm{~d}, 1 \mathrm{H}$, $\mathrm{J}=1.42 \mathrm{~Hz}, \operatorname{Ar}-\mathrm{H}$ ), 7.04 (d, 2H, J=0.72 Hz, Ar-H), 7.00 (d, $2 \mathrm{H}, \mathrm{J}=0.72 \mathrm{~Hz}, \mathrm{Ar}-\mathrm{H}), 6.97(\mathrm{t}, 2 \mathrm{H}, \mathrm{J}=3.69 \mathrm{~Hz}, \mathrm{CHx}-2), 6.95$ $(\mathrm{t}, 2 \mathrm{H}, \mathrm{J}=0.72 \mathrm{~Hz}, \mathrm{CHx}-2), 6.70$ (s, 1H,pyrazole-H), 4.08 (t, $2 \mathrm{H}, \mathrm{J}=3.86 \mathrm{~Hz}, \mathrm{CH}_{2}$, methylene), $3.83(\mathrm{~s}, 1 \mathrm{H}, \mathrm{NH}), 3.81(\mathrm{~s}$, $\left.6 \mathrm{H}, \mathrm{OCH}_{3} \times 2\right), 3.57-2.50(\mathrm{~m}, 8 \mathrm{H}$, morpholine-H), $2.53(\mathrm{t}$, $2 \mathrm{H}, \mathrm{J}=15.53 \mathrm{~Hz}, \mathrm{CH}_{2}$, methylene); ${ }^{13} \mathrm{C}$ NMR $(100 \mathrm{MHz}$, DMSO-d6) $\delta$ ppm:78.90, 78.11, 78.57, 78.24, 66.11, $60.91,60.47,57.99,56.46,53.53,53.32,40.12$, 39.91,
39.70, 39.49, 39.28, 39.07, 38.86, 21.40, 20.61; GC-MS: $281.0 \quad$ (Triazine-Morpholine-M), $355.1 \quad$ (Triazinemorpholine and amine, $\mathrm{M}+2 \mathrm{H}$ ).

\section{4-(2-(1-(4-((4-bromophenyl)amino)-6-(2-morpholino} ethoxy)-1,3,5-triazin-2-yl)-3-(4-hydroxy-3-methoxy styryl)-4,5-dihydro-1Hpyrazol-5-yl)ethyl)-2-methoxy phenol. A4- Dark brown amorphous solid; Yield: 66.07\%; M.P.:84 ${ }^{\circ} \mathrm{C} ;$ FTIR (vmax; $\mathrm{cm}^{-1} \mathrm{KBr}$ ): 3321.49 (O-H stretching), 1567.89 (C=N stretching), $1510.78 \quad\left(\mathrm{CH}_{2}\right.$ bending), 1396.32 (C-Br stretching), 1256.03, 1019.69, 905.06; ${ }^{1} \mathrm{H}$ NMR (400MHz, DMSO-d6, TMS) $\delta$ ppm: 8.19 (s, 2H, Ar-OHx2), 7.44 (d, 2H, J=0.32 Hz,Ar-H), 7.40 (d, $2 \mathrm{H}, \mathrm{J}=0.34 \mathrm{~Hz}, \operatorname{Ar}-\mathrm{H}), 7.38$ (d, $1 \mathrm{H}, \mathrm{J}=0.70 \mathrm{~Hz}, \operatorname{Ar}-\mathrm{H}), 6.82$ (d, $2 \mathrm{H}, \mathrm{J}=0.34 \mathrm{~Hz}, \operatorname{Ar}-\mathrm{H}$ ), 6.80 (d, $2 \mathrm{H}, \mathrm{J}=0.05 \mathrm{~Hz}, \mathrm{Ar}-\mathrm{H}$ ), $6.77(\mathrm{t}, 2 \mathrm{H}, \mathrm{J}=0.32 \mathrm{~Hz}, \mathrm{CHx}-2), 6.74(\mathrm{t}, 2 \mathrm{H}, \mathrm{J}=0.34 \mathrm{~Hz}, \mathrm{CHx}-$ 2), $6.68\left(\mathrm{~s}, 1 \mathrm{H}\right.$, pyrazole-H), $4.10\left(\mathrm{t}, 2 \mathrm{H}, \mathrm{J}=3.86 \mathrm{~Hz}, \mathrm{CH}_{2}\right.$, methylene), $3.92(\mathrm{~s}, 1 \mathrm{H}, \mathrm{NH}), 3.82\left(\mathrm{~s}, 6 \mathrm{H}, \mathrm{OCH}_{3} \times 2\right), 3.58-$ $2.51\left(\mathrm{~m}, 8 \mathrm{H}\right.$, morpholine-H), $2.54\left(\mathrm{t}, 2 \mathrm{H}, \mathrm{J}=3.01 \mathrm{~Hz}, \mathrm{CH}_{2}\right.$, methylene); ${ }^{13} \mathrm{C}$ NMR (100MHz, DMSO-d6) $\delta$ ppm:79.06, 78.73, 78.41, 66.05, 60.49, 57.99, 53.55, 40.17, 39.96, 39.75, 39.51, 39.33, 39.13, 38.92, 21.25; GC-MS: 281.0 (Triazine-Morpholine-M), $417.3 \quad$ (Triazine-morpholine and amine, $\mathrm{M}+3 \mathrm{H}$ ), 647.5 (Curcumin-Para bromoaniline $\mathrm{M}+\mathrm{Cl})$.

4-(2-(1-(4-((3-chlorophenyl)amino)-6-(2-morpholino ethoxy)-1,3,5-triazin-2-yl)-3-(4-hydroxy-3-methoxy styryl)-4,5-dihydro-1Hpyrazol-5-yl)ethyl)-2-methoxy phenol. A5- Dark brown amorphous solid; Yield: 32.67\%; M.P.:80 ${ }^{\circ}$ C; FTIR (vmax; $\mathrm{cm}^{-1} \mathrm{KBr}$ ): $3308(\mathrm{O}-\mathrm{H}$ streching), 2924 (C-H streching), 1643 ( $\mathrm{C}=\mathrm{C}$ streching ), $1395\left(\mathrm{CH}_{3}\right)$, 1395 ( $\mathrm{CH}_{3}$ vibration), 1340 (C-H alkane stretching), 1127, 1021 (C-O streching), 1095 (C-O-C), $905\left(\mathrm{CH}=\mathrm{CH}_{2}\right), 671$ (C-Cl), 538; ${ }^{1} \mathrm{H}$ NMR (400MHz, DMSO, TMS) $\delta$ ppm: 8.19 (s, 2H, Ar-OHx2), 8.04 (s, 1H, Ar-NH), 7.25 (d, 2H, J=1.64 $\mathrm{Hz}, \operatorname{Ar}-\mathrm{H}), 7.23(\mathrm{~d}, 1 \mathrm{H}, \mathrm{J}=2.7 \mathrm{~Hz}, \operatorname{Ar}-\mathrm{H}), 7.22(\mathrm{~d}, 1 \mathrm{H}, \mathrm{J}=2.3$ $\mathrm{Hz}, \operatorname{Ar}-\mathrm{H}), 7.04(\mathrm{~d}, 1 \mathrm{H}, \mathrm{J}=1.7 \mathrm{~Hz}, \operatorname{Ar}-\mathrm{H}), 7.03(\mathrm{~d}, 1 \mathrm{H}, \mathrm{J}=4.6$ $\mathrm{Hz}, \operatorname{Ar}-\mathrm{H}), 7.01(\mathrm{~d}, 1 \mathrm{H}, \mathrm{J}=1.4 \mathrm{~Hz}, \operatorname{Ar}-\mathrm{H}), 6.97(\mathrm{~d}, 1 \mathrm{H}, \mathrm{J}=4.6$ $\mathrm{Hz}, \mathrm{Ar}-\mathrm{H}), 6.95(\mathrm{~d}, 1 \mathrm{H}$, pyrazole-H), $6.78(\mathrm{~d}, 1 \mathrm{H}, \mathrm{J}=1.5 \mathrm{~Hz}$, Ar-H), 6.66 (d, 2H, J=4.6 Hz, Ar-H), $6.63(\mathrm{~d}, 1 \mathrm{H}, \mathrm{J}=1.2 \mathrm{~Hz}$, Ar-H), 4.18 (s, 2H, $\mathrm{CH}_{2}$, Methylene), 3.85-3.76 (s, 6H,2xO $\left.\mathrm{CH}_{3}\right), 2.72\left(\mathrm{~s}, 2 \mathrm{H}, \mathrm{CH}_{2}\right.$, Methylene), 3.57-2.51 (m, 8H, 4x $\mathrm{CH}_{2}$, Morpholine-H), 2.53 (s, $2 \mathrm{H}, \mathrm{CH}_{2}$, Methylene); $13 \mathrm{C}$ NMR (400MHz, DMSO) $\delta$ ppm:79.36, 78.93, 78.73, 78.43, $66.14,59.13,53.62,43.12,39.96,39.75,39.55,39.34$, 
39.13, 38.92; GC-MS: 131.0 (Morpholine-M), 253 (Triazine- amine, $\mathrm{M}+\mathrm{H}$ ), 377 (Curcumin, $\mathrm{M}+\mathrm{Cl}$ ).

\section{4-(2-(5-(4-hydroxy-3-methoxyphenethyl)-1-(4-(2-} morpholinoethoxy)-6-((3nitrophenyl)amino)-1,3,5triazin-2-yl)-4,5-dihydro-1H-pyrazol-3-yl)vinyl)-2methoxyphenol. A6- Dark brown amorphous solid; Yield: 93\%; M.P.:80 ${ }^{\circ}$ C; FTIR (vmax; $\mathrm{cm}^{-1} \mathrm{KBr}$ ): 3372.90 (O-H stretching), 1580.12 ( $\mathrm{C}=\mathrm{N}$ stretching), 1519.89 (NO2 stretching), $\quad 1396.14\left(\mathrm{CH}_{3}\right.$ vibration), 1342.84 (N-N stretching), 1256.92, 1093.49, 905.63; ${ }^{1} \mathrm{H}$ NMR $(400 \mathrm{MHz}$, DMSO-d6, TMS) $\delta$ ppm: 8.19 (s, 2H, Ar-OHx2), 7.51 (d, $1 \mathrm{H}, \mathrm{J}=1.00 \mathrm{~Hz}, \mathrm{Ar}-\mathrm{H}$ ), 7.13 (d, 1H, J=1.17 Hz, Ar-H), 7.12 (d, $1 \mathrm{H}, \mathrm{J}=1.16 \mathrm{~Hz}, \operatorname{Ar}-\mathrm{H}), 7.10(\mathrm{~d}, 1 \mathrm{H}, \mathrm{J}=2.58 \mathrm{~Hz}, \operatorname{Ar}-\mathrm{H})$, pyrazole-H), $6.79(\mathrm{~d}, 2 \mathrm{H}, \mathrm{J}=11.18 \mathrm{~Hz}, \mathrm{Ar}-\mathrm{H}), 6.63(\mathrm{~d}, 2 \mathrm{H}$, $\mathrm{J}=0.86 \mathrm{~Hz}, \operatorname{Ar}-\mathrm{H}), 6.54$ (d, 2H, J=1.16 Hz, Ar-H), 6.53 (t, $4 \mathrm{H}, \mathrm{J}=2.58 \mathrm{~Hz}, \mathrm{CHx}-4), 6.51$ (s, $1 \mathrm{H}$, pyrazole-H), $4.11(\mathrm{t}, 2 \mathrm{H}$, $\left.\mathrm{J}=12.07 \mathrm{~Hz}, \mathrm{CH}_{2}\right), 3.99(\mathrm{~s}, 1 \mathrm{H}, \mathrm{NH}), 3.83\left(\mathrm{~s}, 6 \mathrm{H}, \mathrm{O} \mathrm{CH}_{3} \times 2\right)$, 3.56-2.52 (m, 8H, morpholine-H), $2.55(\mathrm{t}, 2 \mathrm{H}, \mathrm{J}=3.01 \mathrm{~Hz}$, $\left.\mathrm{CH}_{2}\right) ;{ }^{13} \mathrm{C}$ NMR (100MHz, DMSO-d6) $\delta$ ppm:79.06, 78.93, 78.72, 78.04, 66.12, 66.07, 60.50, 58.01, 53.55, 53.34, 40.16, 39.95, 39.74, 39.53, 39.33, 39.12, 38.91, 21.07; GC-MS: 281.0 (Triazine-Morpholine-M), 346.2 (Triazinemorpholine and amine, $\mathrm{M}+\mathrm{H}$ ), 617.3 (Curcumin-meta nitroaniline $\mathrm{M}+\mathrm{Cl}$ ).

\section{4-((4-(3,5-bis(4-hydroxy-3-methoxyphenethyl)-4,5-} dihydro-1Hpyrazol-1-yl)-6-(2 morpholinoethoxy)-1,3,5triazin-2-yl)amino)benzonitrile sulfate hydrate. A7Yellow amorphous solid; Yield: $40 \%$; M.P.:210 $\mathrm{C}$ FTIR (vmax; cm ${ }^{-1} \mathrm{KBr}$ ): 3196.38 (O-H stretching), $3080.37(\mathrm{~N}-\mathrm{H}$ stretching), 2923.38 (Aromatic C-H stretching), 2230.42 $\left(\mathrm{OCH}_{3}\right.$ stretching), $1738.28 \quad(\mathrm{C}=\mathrm{O}$ stretching $), 1683.31$ ( $\mathrm{C}=\mathrm{N}$ stretching), $1586.20\left(\mathrm{CH}_{2}\right.$ bending $), 1551.21(\mathrm{C}=\mathrm{C}$ stretching), 1367.27 ( $\mathrm{N}-\mathrm{N}$ stretching), 779.42; ${ }^{1} \mathrm{H} \mathrm{NMR}$ (400MHz, DMSO-d6, TMS) $\delta$ ppm: 10.84 (s, 2H, Ar-OHx2), $7.46(\mathrm{~d}, 2 \mathrm{H}, \mathrm{J}=0.01 \mathrm{~Hz}, \operatorname{Ar}-\mathrm{H}), 7.32$ (d, $2 \mathrm{H}, \mathrm{J}=0.71 \mathrm{~Hz}, \operatorname{Ar}-$ $\mathrm{H}), 6.81(\mathrm{~d}, 1 \mathrm{H}, \mathrm{J}=0.78 \mathrm{~Hz}$, pyrazole-H), $6.77(\mathrm{~d}, 2 \mathrm{H}, \mathrm{J}=0.01$ $\mathrm{Hz}, \operatorname{Ar}-\mathrm{H}), 6.73(\mathrm{~d}, 2 \mathrm{H}, \mathrm{J}=0.78 \mathrm{~Hz}, \operatorname{Ar}-\mathrm{H}), 6.57$ (d, $2 \mathrm{H}$, $\mathrm{J}=0.72 \mathrm{~Hz}, \operatorname{Ar}-\mathrm{H}), 3.83(\mathrm{~s}, 1 \mathrm{H}, \mathrm{NH}), 3.68\left(\mathrm{~s}, 6 \mathrm{H}, \mathrm{OCH}_{3} \times 2\right)$, $3.60\left(\mathrm{t}, 2 \mathrm{H}, \mathrm{J}=3.47 \mathrm{~Hz}, \mathrm{CH}_{2}\right.$, methylene), $3.58(\mathrm{~d}, 4 \mathrm{H}$, $\mathrm{J}=0.14 \mathrm{~Hz}$, morpholine- $\mathrm{H}), 2.51-1.91(\mathrm{~m}, 4 \mathrm{H}$, aliphatic $\mathrm{CH} 4$ ), 2.49 (t, $2 \mathrm{H}, \mathrm{J}=3.60 \mathrm{~Hz}, \mathrm{CH}_{2}$, methylene), 2.50 (d, $4 \mathrm{H}, \mathrm{J}=3.60 \mathrm{~Hz}$, morpholine-H); ${ }^{13} \mathrm{C}$ NMR (100MHz, DMSOd6) $\delta$ ppm:172.07, 165.52, 164.39, 161.36, 148.19, $133.07,132.73,124.07,120.57,119.04,113.28,108.87$, $108.73,107.86,104.95,40.03,39.82,39.62,39.41$,
39.20, 38.99, 38.78, 21.40, 21.04; GC-MS: 281.0 (Triazine-Morpholine-M), 326.5 (Triazine- morpholine and amine, $\mathrm{M}+\mathrm{H}$ ).

\section{4-(2-(1-(4-((2-chlorophenyl)amino)-6-(2-morpholino ethoxy)-1,3,5-triazin-2-yl)-3-(4-hydroxy-3-metho xystyryl)-4,5-dihydro-1Hpyrazol-5-yl)ethyl)-2-methoxy} phenol. A8- Dark brown amorphous solid; Yield: $81.15 \%$; M.P.:150 ${ }^{\circ} \mathrm{C}$; FTIR (vmax; $\mathrm{cm}^{-1} \mathrm{KBr}$ ): 3371.84 (O-H stretching), 1562.62 (C=N stretching), $1393.29 \quad\left(\mathrm{CH}_{3}\right.$ vibration), 1260.51 (N-N stretching), 1125.11, 1092.48, 749.94 (C-Cl stretching); ${ }^{1} \mathrm{H}$ NMR (400MHz, DMSO-d6, TMS) $\delta$ ppm: 8.17 (s, 2H, Ar-OHx2), 7.44 (d, 1H, J=0.76 $\mathrm{Hz}, \mathrm{Ar}-\mathrm{H}), 7.42$ (d, 1H, J=0.89 Hz, Ar-H), 7.32 (d, 1H, J=1.36 $\mathrm{Hz}, \operatorname{Ar}-\mathrm{H}), 7.31$ (d, $1 \mathrm{H}, \mathrm{J}=0.89 \mathrm{~Hz}, \operatorname{Ar}-\mathrm{H}), 7.16$ (d, 2H, $\mathrm{J}=5.19 \mathrm{~Hz}, \operatorname{Ar}-\mathrm{H}), 7.11$ (d, 2H, J=1.36 Hz, Ar-H), 6.79 (d, $2 \mathrm{H}, \mathrm{J}=0.89 \mathrm{~Hz}, \mathrm{Ar}-\mathrm{H}), 6.76-6.67(\mathrm{t}, 4 \mathrm{H}, \mathrm{J}=5.19 \mathrm{~Hz}, \mathrm{CHx}-4)$, $6.63\left(\mathrm{~s}, 1 \mathrm{H}\right.$,pyrazole-H), $4.11\left(\mathrm{t}, 2 \mathrm{H}, \mathrm{J}=5.24 \mathrm{~Hz}, \mathrm{CH}_{2}\right), 3.86$ $(\mathrm{s}, 1 \mathrm{H}, \mathrm{NH}), 3.82\left(\mathrm{~s}, 6 \mathrm{H}, \mathrm{OCH}_{3} \times 2\right), 3.57-2.52(\mathrm{~m}, 8 \mathrm{H}$, morpholine-H), $2.56\left(\mathrm{t}, 2 \mathrm{H}, \mathrm{J}=11.38 \mathrm{~Hz}, \mathrm{CH}_{2}\right) ;{ }^{13} \mathrm{C} \mathrm{NMR}$ (100MHz, DMSO-d6) $\delta$ ppm: 129.07, 127.05, 79.02, 78.89, 78.69, 78.36, 66.07, 60.50, 58.00, 53.55, 53.34, $40.17,39.96,39.75,39.54,39.33,39.12,38.92,21.42$; GC-MS: $\quad 282.7 \quad$ (Triazine-Morpholine-M+H), 601.2 (Curcumin-Para chloroaniline $\mathrm{M}+\mathrm{Cl}$ ), $697.2(\mathrm{M})$.

\section{4-(2-(5-(4-hydroxy-3-methoxyphenethyl)-1-(4-(2-} morpholinoethoxy)-6-((4 nitrophenyl)amino)-1,3,5triazin-2-yl)-4,5-dihydro-1H-pyrazol-3-yl)vinyl)-2-

methoxyphenol. A9- Dark brown amorphous solid; Yield: 71\%; M.P.:60 ${ }^{\circ} \mathrm{C}$; FTIR (vmax; $\mathrm{cm}^{-1} \mathrm{KBr}$ ): $3246.17(\mathrm{O}-\mathrm{H}$ stretching), 2933.11 (C-H stretching), 1729.71 (C=O stretching), 1568.63 ( $\mathrm{C}=\mathrm{N}$ stretching), 1498.61 (NO2 stretching), $1378.73 \quad\left(\mathrm{CH}_{3}\right.$ vibration), 1323.84 (N-N stretching), 1253.52, 1023.08, 905.28; ${ }^{1} \mathrm{H} \mathrm{NMR}(400 \mathrm{MHz}$, DMSO-d6, TMS) $\delta$ ppm: 8.17 (s, 2H, Ar-OHx2), 8.06 (d, $2 \mathrm{H}, \mathrm{J}=4.91 \mathrm{~Hz}, \mathrm{Ar}-\mathrm{H}$ ), 7.53 (d, 2H, J=1.27 Hz, Ar-H), 7.18 (d, $2 \mathrm{H}, \mathrm{J}=2.62 \mathrm{~Hz}, \operatorname{Ar}-\mathrm{H}), 7.09$ (d, 2H, J=2.61 Hz, Ar-H), 7.00 (d, $2 \mathrm{H}, \mathrm{J}=1.27 \mathrm{~Hz}, \mathrm{Ar}-\mathrm{H}), 6.97$ (t, $2 \mathrm{H}, \mathrm{J}=4.25 \mathrm{~Hz}, \mathrm{CH} \times 2$ ), $6.95(\mathrm{t}, 2 \mathrm{H}, \mathrm{J}=2.62 \mathrm{~Hz}, \mathrm{CH} \times 2), 6.67(\mathrm{~s}, 1 \mathrm{H}$, pyrazole-H), $4.34\left(\mathrm{t}, 2 \mathrm{H}, \mathrm{J}=2.21 \mathrm{~Hz}, \mathrm{CH}_{2}\right), 3.92(\mathrm{~s}, 1 \mathrm{H}, \mathrm{NH}), 3.84(\mathrm{~s}, 6 \mathrm{H}$, $\left.\mathrm{OCH}_{3} \times 2\right), 3.70-2.55(\mathrm{~m}, 8 \mathrm{H}$, morpholine-H), $2.44(\mathrm{t}, 2 \mathrm{H}$, $\left.\mathrm{J}=3.85 \mathrm{~Hz}, \mathrm{CH}_{2}\right) ;{ }^{13} \mathrm{C}$ NMR (100MHz, DMSO-d6) $\delta$ ppm:78.81, 78.48, 78.16, 39.87, 39.67, 39.46; GC-MS: 281.1 (Triazine-Morpholine-M), $345.2 \quad$ (Triazinemorpholine and amine, $\mathrm{M}$ ). 
4-(2-(5-(4-hydroxy-3-methoxyphenethyl)-1-(4-(2morpholinoethoxy)-6-(phenylamino)-1,3,5-triazin-2-yl)4,5-dihydro-1H-pyrazol-3-yl)vinyl)-2-methoxyphenol.

A10- Dark brown amorphous solid; Yield: 68.49\%; M.P.:160 ${ }^{\circ}$ C;FTIR (vmax; $\quad \mathrm{cm}^{-1} \mathrm{KBr}$ ): $3032.76 \quad$ (C-H stretching), 1720.60 ( $\mathrm{C}=\mathrm{O}$ stretching), $1551.29 \quad(\mathrm{C}=\mathrm{N}$ stretching), $1389.86 \quad\left(\mathrm{CH}_{3}\right.$ vibration), $1324.82 \quad(\mathrm{~N}-\mathrm{N}$ stretching), 1264.18, 1022.77, 958.75; ${ }^{1} \mathrm{H}$ NMR $(400 \mathrm{MHz}$, DMSO-d6, TMS) $\delta$ ppm: 9.65 (s, 2H, Ar-OHx2), 7.62 (d, $2 \mathrm{H}, \mathrm{J}=1.10 \mathrm{~Hz}, \mathrm{Ar}-\mathrm{H}$ ), 7.31 (d, 2H, J=4.65 Hz, Ar-H), 7.14 (d, $2 \mathrm{H}, \mathrm{J}=1.87 \mathrm{~Hz}, \mathrm{Ar}-\mathrm{H}), 7.10(\mathrm{~d}, 2 \mathrm{H}, \mathrm{J}=5.70 \mathrm{~Hz}, \mathrm{Ar}-\mathrm{H}), 7.07$ (d, $1 \mathrm{H}, \mathrm{J}=5.06 \mathrm{~Hz}, \mathrm{Ar}-\mathrm{H}$ ), 6.87 (d, 2H, J=2.26 Hz, Ar-H), $6.86(\mathrm{t}, 2 \mathrm{H}, \mathrm{J}=2.17 \mathrm{~Hz}, \mathrm{CHx}$ ), $6.84(\mathrm{t}, 2 \mathrm{H}, \mathrm{J}=1.09 \mathrm{~Hz}$, $\mathrm{CH} 2$ ), $6.68(\mathrm{~s}, 1 \mathrm{H}$, pyrazole-H), $5.87(\mathrm{t}, 2 \mathrm{H}, \mathrm{J}=1.87 \mathrm{~Hz}$, $\left.\mathrm{CH}_{2}\right), 3.84(\mathrm{~s}, 1 \mathrm{H}, \mathrm{NH}), 3.35\left(\mathrm{~s}, 6 \mathrm{H}, \mathrm{OCH}_{3} \times 2\right), 3.56-2.49(\mathrm{~m}$, $8 \mathrm{H}$, morpholine-H), $2.51\left(\mathrm{t}, 2 \mathrm{H}, \mathrm{J}=1.45 \mathrm{~Hz}, \mathrm{CH}_{2}\right) ;{ }^{13} \mathrm{C} \mathrm{NMR}$ (100MHz, DMSO-d6) $\delta$ ppm:183.17, 165.57, 164.42, $161.40,154.21,149.33,148.23,147.96,140.66,137.28$, $128.74,126.30,124.11,121.17,121.06,115.67,111.32$,
107.93, 55.67, 40.12, 39.91, 39.08, 38.87, 21.48; GC-MS: 281.1 (Triazine-Morpholine-M), $537.0 \quad$ (Curcumintriazine-aniline $\mathrm{M}+{ }^{3} \mathrm{H}$ ).

Antibacterial activity of synthesized curcumin derivatives- The antibacterial activity of ten curcumin pyrazole triazine conjugates was evaluated and the screening results are reported in Table 1 . It was evident from the assay that compound 5 and 8 both have chlorine atom in their aniline moiety enhanced their antimicrobial activity. In the case of the most potent drug $A 5$ it has been reported that among gram-positive bacteria $B$. subtilis, S. aureus and Clostridium perfringens were found to have the highest zone of inhibition ranged 27. Total $26 \mathrm{~mm}$ and $25 \mathrm{~mm}$, among gram-negative bacteria drug was found most potent against $P$. aeruginosa with a zone of inhibition of $28 \mathrm{~mm}$. The overall efficacy of different compounds can be found as $5>8>3>4>6>7>10>1>2>9$.

Table 1: Antibacterial activity of curcumin pyrazole triazine conjugates

\begin{tabular}{|c|c|c|c|c|c|c|c|c|c|c|c|}
\hline \multirow{2}{*}{$\begin{array}{l}\text { S. } \\
\text { No. }\end{array}$} & \multirow{2}{*}{ Test organisms } & \multicolumn{10}{|c|}{ Zone of inhibition (mm) } \\
\hline & & A1 & A2 & A3 & A4 & A5 & A6 & A7 & A8 & A9 & A10 \\
\hline 1 & L. monocytogenes & 5.66 & 7.33 & 14.66 & 14.66 & 13.66 & 15.00 & 6.33 & 14.00 & 7 & 8.33 \\
\hline 2 & B. cereus & 8.66 & 8.33 & 13.33 & 11.33 & 12.33 & 10.33 & 11.33 & 20.33 & 7.33 & 10.33 \\
\hline 3 & B. subtilis & 8.33 & 10.33 & 15.66 & 27.66 & 26.33 & 22.66 & 17.66 & 26.67 & 7.33 & 9.00 \\
\hline 4 & C. perfringens & 11.00 & 10 & 19.66 & 16.66 & 24.66 & 20.66 & 11.33 & 15.67 & 9.66 & 11.67 \\
\hline 5 & S. aureus & 7.00 & 8.33 & 31.66 & 27.66 & 26.33 & 23.66 & 13.66 & 31.33 & 7.66 & 10.33 \\
\hline 6 & S. pyogenes & 5.66 & 6.33 & 6.66 & 0 & 11.33 & 0 & 8.00 & 13.33 & 0 & 6.33 \\
\hline 7 & E. coli & 6.00 & 6 & 20.00 & 18.66 & 17.66 & 16.66 & 11.66 & 14.67 & 6.33 & 6.00 \\
\hline 8 & P. aeruginosa & 0 & 0 & 22.66 & 26.66 & 27.66 & 25.66 & 11.66 & 25.67 & 0 & 7.00 \\
\hline 9 & S. typhi & 6.33 & 6.33 & 14.33 & 16.66 & 14.66 & 17.00 & 11.33 & 17.67 & 6 & 6.00 \\
\hline 10 & S. dysenteriae & 6.33 & 6.66 & 15.66 & 16.00 & 15.33 & 16.33 & 11.66 & 17.33 & 7.66 & 6.66 \\
\hline 11 & V. cholera & 6.33 & 6.66 & 15.33 & 15.00 & 15.66 & 11.66 & 11.66 & 17.67 & 7.66 & 6.66 \\
\hline 12 & C. jejuni & 6.66 & 6.33 & 12.33 & 12.33 & 12.33 & 11.33 & 0 & 16.00 & 0 & 6.00 \\
\hline 13 & H. pylori & 6.33 & 0 & 11.33 & 11.66 & 14.66 & 12.00 & 6.00 & 13.33 & 7.00 & 10.33 \\
\hline
\end{tabular}

* includes well size of $5 \mathrm{~mm}$ diameter

A1=4-aminobenzonitrile, A 2=4-aminobenzonitrile, A3= 3-floroaniline, A 4=4-bromoaniline, A5=3-cloroaniline, A6= 3-nitroaniline, A7 = 2-nitroaniline, $A 8=2$-cloroaniline, $A 9=4$-nitroaniline, $10=$ Aniline 
Minimum inhibitory concentration and Minimum Bactericidal concentration- The minimum inhibitory concentration of synthesized Curcumin pyrazole triazine conjugate A5 having a best antimicrobial activity was determined as depicted in Table 2 by resazurin based micro broth dilution method. The drugs were taken in the concentration ranges as 512, 256, 128, 64 and 32 $\mu \mathrm{g} / \mathrm{ml}$. Among gram-negative bacteria, the best MIC was observed in the case of $P$. aeruginosa $32 \mu \mathrm{g} / \mathrm{ml}$ as compared to $H$. pylori $64 \mu \mathrm{g} / \mathrm{ml}$ and E. coli, S. dysentriae with MIC value $128 \mu \mathrm{g} / \mathrm{ml}$. Among Gram-positive bacteria, $S$. aureus was found to have the lowest MIC that is $32 \mu \mathrm{g} / \mathrm{ml}$ than $B$. subtilis and L. monocytogenes showed MIC at $64 \mu \mathrm{g} / \mathrm{ml}$ and the highest value 128 $\mu \mathrm{g} / \mathrm{ml}$ was observed in the case of $C$. perfringens. Both gram-negative bacteria $P$. aeruginosa and gram-positive bacteria $S$. aureus were found to have good minimum bactericidal activity at $128 \mu \mathrm{g} / \mathrm{ml}$ concentration. Except for $E$. coli with an $\mathrm{MBC}$ value of $512 \mu \mathrm{g} / \mathrm{ml}$ all microbes such as $B$. subtilis, $S$. dysentriae, L. monocytogenes and $H$. pylori reported with highest $\mathrm{MBC}$ value of concentration $256 \mu \mathrm{g} / \mathrm{ml}$.

Table 2: MIC and MBC of bacterial pathogens

\begin{tabular}{cccc}
\hline S.No. & Organisms & $\begin{array}{c}\text { MIC } \\
(\mu \mathrm{g} / \mathrm{ml})\end{array}$ & $\begin{array}{c}\text { MBC } \\
(\mu \mathrm{g} / \mathrm{ml})\end{array}$ \\
\hline 1 & E. coli & 128 & 512 \\
2 & S. aureus & 32 & 128 \\
3 & B. subtilis & 64 & 256 \\
4 & S. dysenteriae & 128 & 256 \\
5 & C. perfringens & 128 & 256 \\
6 & L. monocytogenes & 64 & 256 \\
7 & H. pylori & 64 & 256 \\
8 & P. aeruginosa & 32 & 128 \\
\hline
\end{tabular}

Antifungal activity of synthesized curcumin derivativesVaried curcumin pyrazole triazine conjugates were tested for antifungal activity using the agar well diffusion process, shown in Table 3 in accordance with CLSI. All synthesized compounds were found to be resistant to the pathogenic mold $A$. fumigatus. Only four drugs displayed antifungal efficacy against pathogenic yeast Candida albicans with compound A5 having the most potent zone of inhibition $(21 \mathrm{~mm})$ and compound A4 having the least potent zone of inhibition $(14 \mathrm{~mm})$. As a consequence of the findings, chlorine-containing aniline derivatives were discovered to be the most potent antifungal agent. The presence of a chlorine atom in meta-position increased its antifungal effect. In comparison to these halogens, fluorine at ortho-position and bromine at para-position yielded important effects, but not as much as the chlorine-containing aniline derivatives of curcumin pyrazole triazine conjugates.

Table 3: Antifungal activity of curcumin pyrazole triazine conjugates

\begin{tabular}{ccc}
\hline \multirow{2}{*}{ Compounds } & \multicolumn{2}{c}{ Zone of inhibition $(\mathbf{m m})^{*}$} \\
\cline { 2 - 3 } & C. albicans & A. fumigatus \\
\hline A1 & 0 & 0 \\
A2 & 0 & 0 \\
A3 & 15 & 0 \\
A4 & 14 & 0 \\
A5 & 21 & 0 \\
A6 & 0 & 0 \\
A7 & 0 & 0 \\
A8 & 17 & 0 \\
A9 & 0 & 0 \\
A10 & 0 & 0 \\
\hline
\end{tabular}

* includes the well size of $5 \mathrm{~mm}$ diameter

A1=4-aminobenzonitrile, A $2=4$-aminobenzonitrile, $\quad A 3=3$ floroaniline, A $4=4$-bromoaniline, $A 5=3$-cloroaniline, $A 6=3$ nitroaniline, $A 7=2$-nitroaniline, $A 8=2$-cloroaniline, $A 9=4$ nitroaniline, $10=$ Aniline

\section{DISCUSSION}

Concerned about the ongoing emergence of multidrugresistant bacterial and fungal disease, there is an urgent need for the development of new antimicrobial agents [22,23]. Even newly developed antibiotics are ineffective against these MDRs ${ }^{[24]}$. In this regard, we proposed unique curcumin pyrazole triazine conjugates that have been found to have antibacterial and antifungal action. Nitrogen-containing heterocyclic compounds with higher binding affinities to biological receptors have piqued the interest of many researchers as compounds to use as conjugating moieties ${ }^{[25]}$. As a result of its increased importance in biological studies, particularly for antibacterial ${ }^{[26]}$ and antifungal activity ${ }^{[19,27]}$. A nitrogencontaining heterocyclic compound with good biological activities known as 2, 4, 6-trichloro-1, 3, 5-triazine has been used as a conjugating agent with curcumin. For verification of synthesized curcumin pyrazole triazine conjugates, their structures were characterized by different spectroscopic methods such as FTIR, FT NMR $\left({ }^{1} \mathrm{H} \quad \mathrm{NMR}\right.$ and ${ }^{13} \mathrm{C}$ NMR) and Mass Spectrometry. Thereafter synthesized curcumin pyrazole triazine conjugates were evaluated for the antibacterial and 
antifungal activity that resulted in significant outcomes against both fungal and bacterial pathogens.

Against both types of pathogens compound A5 containing 3-chloroaniline moiety was found to possess the most potent antimicrobial activity. Among six grampositive bacteria only four $L$. monocytogenes, S. aureus, $B$. subtilis and $C$. perfringens were found to be most susceptible to compound $A 5$ having a zone of inhibition 14, 26, 27, and $25 \mathrm{~mm}$ and MIC values of $64,32,64$ and $128 \mu \mathrm{g} / \mathrm{ml}$. Among seven gram-negative bacterial E. coli, $S$. dysenteriae, H. pylori, P. aeruginosa was reported with the best zone of inhibition 18,16,15, and $28 \mathrm{~mm}$ and MIC values with 128, 128, 64 and $32 \mu \mathrm{g} / \mathrm{ml}$. Regarding antifungal agent similar compound A5 was found to be the most potent against $C$. albicans with zone of inhibition of $21 \mathrm{~mm}$. All synthesized compounds were found to be resistant to the pathogenic $A$. fumigatus. According to these findings, curcumin pyrazole triazine conjugates containing halogen derived anilines such as $\mathrm{Cl}, \mathrm{Br}$, and $\mathrm{F}$ can bear a biologically significant hybrid molecule, which could address a lead compound for various targets and may provide the possibility of reducing multidrug resistance.

\section{CONCLUSIONS}

Novel Curcumin pyrazole triazine conjugates were synthesized bearing covalent linkage with suitable ligands to enhance its biological activity and characterized by the FTIR ${ }^{1} \mathrm{H}$ NMR, ${ }^{13} \mathrm{C}$ NMR and Mass spectrometry. The investigation of antibacterial and antifungal screening data revealed that all the Curcumin derivatives bearing halogen moiety showed moderate to good bacterial and fungal inhibition. Significant results were obtained in the case of both gram-positive bacteria Bacillus subtilis with the zone of inhibition $28 \mathrm{~mm}$ and MIC $32 \mu \mathrm{g} / \mathrm{ml}$ and gram-negative bacteria Pseudomonas aeruginosa with the zone of inhibition $28 \mathrm{~mm}$ and MIC $32 \mu \mathrm{g} / \mathrm{ml}$. Among pathogenic fungi, Candida albicans was found to be most susceptible to compound A5.

The pharmaceutically important hybrid molecules can be used in future to cure bacterial and fungal diseases.

\section{ACKNOWLEDGMENTS}

The authors are thankful to the Department of Pharmaceutical Sciences and the Department of Industrial Microbiology, Sam Higginbottom University of Agriculture, Technology and Sciences for providing necessary research facilities to carrying out experiments.

\section{CONTRIBUTION OF AUTHORS}

Research concept- Dr. Ebenezer Jeyakumar, Dr. Rubina Lawrence

Research design- Dr. Ebenezer Jeyakumar, Dr. Uday

Pratap Singh

Supervision- Dr. Ebenezer Jeyakumar

Materials-Anjali

Data collection-Anjali

Data analysis and Interpretation- Dr. Uday Pratap Singh, Anjali

Literature search- Anjali

Writing article- Anjali

Critical review- Dr. P. Malairajan

Article editing- Anjali

Final approval- Dr. Ebenezer Jeyakumar

\section{REFERENCES}

[1] Tanwar J, Das S, Fatima Z, Hameed S. Multidrug Resistance: An Emerging Crisis. Inter Pers Inf Dis., 2014; pp. 1-7.

[2] Roca I, Akova M, Baquero F, Carlet J, Cavaleri M, et al. The global threat of antimicrobial resistance: science for intervention. New Microbes New Infect., 2015; 16(6): 22-9.

[3] Noval M, Banoub M, Claeys KC, Heil E. The Battle Is on: New Beta-Lactams for the Treatment of Multidrug-Resistant Gram-Negative Organisms. Cur Infect Dis Rep., 2020; 22(1): 1.

[4] Reddy P, Chadaga S, Noskin GA. Antibiotic Considerations in the Treatment of MultidrugResistant (MDR) Pathogens: A Case-Based Review. J Hosp Med., 2009; 4(6): E8-15.

[5] Nguyen L, Garcia J, Gruenberg K, Conan, MacDougall. Multidrug-Resistant Pseudomonas Infections: Hard to Treat, But Hope on the Horizon?. Curr Infect Dis Rep., 2018; 20(8): 23.

[6] Kumar A, Jaitak V. Natural products as multidrug resistance modulators in cancer. Eur J Med Chem., 2019; 176: 268-91.

[7] Su T, Qiu Y, Hua X, Ye B, Luo $H$, et al. Novel opportunity to Reverse antibiotic resistance: To explore traditional chinese medicine with potential activity against antibiotics-resistance bacteria. Front Microbiol., 2020; 11: 61-70.

[8] Chew J, Peh S, Yeang TS. Non-microbial Natural Products That Inhibit Drug- Resistant Staphylococcus aureus. Intechopen., 2018; pp. 1-31. 
[9] Corta A, Ozbena T. Natural product modulators to overcome multidrug resistance in cancer. Nutr Cancer, 2015; pp. 1-13.

[10]Khor P Y, Aluwi MFFM, Rullah K, Lam KW. Insights on the synthesis of asymmetric curcumin derivatives and their biological activities. Eur J Med Chem., 2019; 1; 183: 111704.

[11]Hewlings DJ, Kalman DS. Curcumin: A Review of Its' Effects on Human Health. Foods, 2017; 6(10): 92.

[12]Lahlou M. The Success of Natural Products in Drug Discovery. J Pharm Pharmcol., 2013; 4: 17-31.

[13]Aggarwal BB, Gupta SC, Sung B. Curcumin: an orally bioavailable blocker of TNF and other proinflammatory biomarkers. Br J Pharmcol., 2013; 169: 1672-92.

[14]Carlos AM, Nano MLT, Rosatella AA. Synthesis of 2, 4, 6-trisubstituted 1, 3, 5-triazines. Mole., 2006; 81102.

[15]Sarmah KN, Sarmah NK, Kurmi KB, Patel TV. Synthesis of novel derivatives containing s-triazine moiety as potential antibacterial agents. Arch Appl Sci Res., 2012; 4: 805-08.

[16]Bhat HR, Pandey Pk, Ghosh SK, Singh UP. Development of 4-aminoquinolone-1, 3, 5-triazine conjugates as potent antibacterial agent through facile synthetic route. Med Chem Res., 2013; 28: 816.

[17]Al-Khodir FAI, Al-Warti T, Abumelha HMA, Al-Issa SA. Synthesis, chemical and biological investigations of new $\mathrm{Ru}(\mathrm{III})$ and $\mathrm{Se}(\mathrm{IV})$ complexes containing 1,3,5triazine chelating derivatives. J Mol Struct., 2018; 795-808.

[18]Solankee A, Kapadia K, Sokovic AM, Dotchinova I, Geronikaki A. Synthesis of some new s-triazine based chalcones and their derivatives as potent antimicrobial agents. Eur J Med chem., 2010; 510-18.
[19]Singh UP, Pathak $M$, Dubey $V$, et al. Design, synthesis, antibacterial activity and molecular docking studies of novel hybrids 1, 3-thiazine-1, 3, 5triazine derivatives as bacterial translational inhibitor. Chem Biol Drug Des., 2012; 80: 572-583.

[20]Andrews J. Determination of minimum inhibitory concentrations. J Antimicrobe Chemotherap., 2001; 48: 5-16.

[21]Mariita R, Ogol C, Oguge N, Okeno P. Methanol extract of three medicinal plants from Samburu in Northern Kenya show significant antimycobacterial, antibacterial and antifungal properties. J Med Plant Res., 2011; 5(1): 54-64.

[22]Srivastava P, Shukla M, Kaul G, Chopra S, Patra AK. Rationally designed curcumin based ruthenium (II) antimicrobials effective against drug-resistant Staphylococcus aureus. Dalton Trans., 2019; 48: 11822-28.

[23]Vivas R, Andrea A, Barbosa T, Santana S, Dolabela, Jain S. Multidrug-Resistant Bacteria and Alternative Methods to Control Them: An Overview. Microbe Drug Resist., 2019; 25(6): 890-908.

[24]Che CT, Zhang H. Plant Natural Products for Human Health. Int J Mol Sci., 2019; (4): 830.

[25]Desai NC, Malwana AH, et al. Synthesis, characteri zation and antimicrobial activity of some new4(40(2-isoniocotinoyl hydrazinyl)-6-((aryl) amino)1,3,5-triazin-2-ylamino)- $\mathrm{N}$-(pyrimidin-2-yl) benzene sulfonamides. Indian J Chem., 2013; 5(1): 21-7.

[26]Patel PK, Patel RV, Mahajan DH, Parikh PA, Mehta $\mathrm{GN}$, et al. Design, synthesis, characterization and in vitro antimicrobial activity of novel trisubstituted striazines. Med Chem Res., 2012; 3182-94.

[27]Duan Y, Li K, Wang H, Wu T, Zhao Y, et al. Preparation and evaluation of Curcumin grafted hyaluronic acid modified pollan polymers as a functional wound dressing material. Carbohydr Polym., 2020; 238: 116195. 